\title{
Aspergillus spinal epidural abscess: case presentation and review of the literature
}

\author{
Faris Shweikeh ${ }^{1,2} \cdot$ Stephanie Zyck $^{3} \cdot$ Fadi Sweiss $^{4} \cdot$ Ajleeta Sangtani $^{1} \cdot$ Mohammed Shweikeh $^{5} \cdot$ Husam Issa $^{6}$. \\ Michael P. Steinmetz ${ }^{7} \cdot$ Georges Z. Markarian ${ }^{1}$
}

Received: 7 July 2017 / Revised: 8 January 2018 / Accepted: 11 January 2018

(c) International Spinal Cord Society 2018

\begin{abstract}
Study design In this review, we present a case of Aspergillus spinal epidural abscess (ASEA) and review the literature. Objectives To provide further insight on a rare condition.

Setting A description of a patient with ASEA in a 58-year-old woman that was successfully treated with conservative management is presented.

Methods Following case presentation, a literature search (MedLine and PubMed) and assessment of epidemiology, presentation, diagnosis, treatments, and outcomes is performed.

Results Review of the literature finds 26 reported cases. The infection occurs in males with a higher frequency (66.7\%). The thoracic and lumbar regions are more likely afflicted (96.1\%). Common symptoms are backache, neurological deficits, and fever. Most frequent comorbidities were malignancy, diabetes mellitus, and immunodeficiency. Complications were numerous and often catastrophic. Treatment entailed a combination of antibiotics and surgery. Overall, ASEA patients did poorly: death in majority (52\%), minimal recovery in $22 \%$, and others did attain full recovery (26\%).

Conclusions Generally, this infection has high morbidity and mortality. Early identification is important to a successful outcome. Appropriate management with antifungals is central and proves to be effective as seen in the reported case though surgical intervention is usually a necessity as the literature suggests. From an epidemiological and public health perspective, particularly with recent outbreaks, understanding the treatment of this rare CNS infection becomes even more imperative.
\end{abstract}

Faris Shweikeh

faris.shweikeh@gmail.com

1 Department of Neurosurgery, Cedars-Sinai Medical Center, Los Angeles, CA, USA

2 Summa Health System, College of Medicine, Northeast Ohio Medical University, Rootstown, OH, USA

3 Department of Neurosurgery, SUNY Upstate Medical University, Syracuse, NY, USA

4 Department of Neurological Surgery, George Washington University, Washington, DC, USA

5 Family Medicine Center, St. Joseph's Hospital and Medical Center, Phoenix, AZ, USA

6 Memorial Hermann Hospital, University of Texas Health Science Center, Houston, TX, USA

7 Center for Spine Health, Department of Neurosurgery, Cleveland Clinic, Cleveland, OH, USA

\section{Introduction}

The fungus of the genus Aspergillus is an inhaled pathogen in humans that can result in various forms of pulmonary diseases [1]. It primarily effects those with chronic lung disease or who are immunocompromised. In some cases, the fungus may invade other loci outside of the lungs, occurring either contiguously or hematogenously. Invasive aspergillosis rarely affects bone structures, but when it does occur, the spine is the most common location [2-4]. Aspergillus infection of the spine can sequentially result in a spinal epidural abscess, typically occurring in young patients [3, 4]. Aspergillus spinal epidural abscess (ASEA) usually occurs via hematogenous spread from a respiratory focus, gastrointestinal focus, sinuses, or via contiguous spread following surgery or procedures in the same area $[2$, 5-9]. ASEA typically occurs in the thoracic or lumbar spine, but has also been reported in the cervical spine $[2,8$, 9]. 
As the rate of immunocompromised status among the general populace increases, the occurrence of fungal infections observes a proportional rise in prevalence [1012]. In addition, fungal infections have been of epidemiological interest as their rarity yet increasing prevalence brings about an awareness in all of its aspects including pathophysiology and management. For instance, recently there was an outbreak of central nervous system fungal infections among patients receiving epidural or paraspinal glucocorticoid injections in multiple regions in the United States [13-15]. Patients reportedly developed fungal meningitis, spinal osteomyelitis, and epidural abscess. Multiple patients had confirmatory fungal infections, including cases with Aspergillus species.

Given the rarity of the condition, only a few patients with ASEA have been reported in the medical literature. Here, we present a fascinating case of ASEA that was successfully treated nonsurgically. We also review the current literature and provide a summary of clinical factors including risks, treatments and outcome.

\section{Methods}

A case of a patient with an ASEA is presented followed by a review of the relevant literature. For the literature search, a query of the PubMed and MEDLINE databases was performed to identify articles reporting on ASEA. The Preferred Reporting Items for Systematically Reviews and Meta-Analyses (PRISMA) was utilized as a guide (Fig. 1).

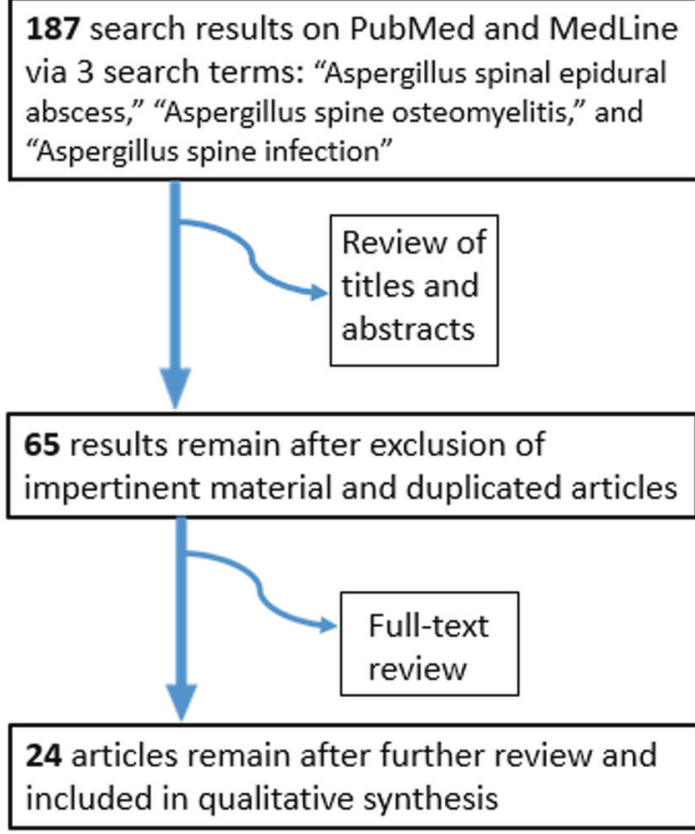

Fig. 1 Details of the literature search strategy using PRISMA guidelines
The search terms "Aspergillus spinal epidural abscess," "Aspergillus spine osteomyelitis," and "Aspergillus spine infection" were used and returned 187 results. These articles were then examined for relevant cases describing the infection. In addition, the reference lists from these articles were also inspected for other relevant articles. Only publications in English, peer-reviewed journals were selected. The criteria for inclusion into the review was: the article had to report demographic characteristics, clinical presentation, diagnostic information, and discussed treatment, complications, and outcomes at follow-up of patients with ASEA. In addition, articles that described spinal infections without frank epidural abscess, a different spinal disease entity, or lacked relevance were excluded. From the original search, 65 articles remained after exclusion of irrelevant content and duplicated material. Following removal of records that did not meet the inclusion criteria, the remaining 24 were included in this review. Articles were reviewed with an emphasis on predisposing factors, diagnosis, successful treatments, and prognosis. Microsoft Excel (Microsoft Corporation, Redmond, WA) was utilized for entry of data and its analysis.

\section{Results}

\section{Case Presentation}

The patient is a 58-year-old woman with a past medical history of severe chronic obstructive pulmonary disease, chronic lower back pain, multiple back surgeries, and steroid epidural injections. She was also on chronic treatment with oral prednisone. She presented with a month long history of back pain and radicular symptoms. Patient had been seen a month prior by her orthopedist and diagnosed with mild-moderate L4-L5 foraminal stenosis. MRI done at that time was consistent with spinal stenosis without any evidence of other pathology, and she was treated medically with non-steroidal anti-inflammatory medication.

She presented to the emergency department for increasing and persistent back pain and new onset fevers and chills. She was found to be tachycardic but afebrile, with lumbar paraspinal and spinal tenderness as well as tenderness over the right thigh and leg. Her strength, sensation, and reflexes were within normal limits. She had normal rectal tone. Complete blood count was negative for leukocytosis or bandemia. Erythrocyte sedimentation rate was elevated at 91. Emergent MRI was indicative of an L4-S1 epidural abscess, diskitis and osteomyelitis (Fig. 2). In addition, she was found to have evidence of septic arthritis in the right sacroiliac joint with an additional small abscess anteriorly.

The patient was admitted and started on empiric treatment with antibiotics (Vancomycin and Clindamycin) for 
Fig. 2 MRI scans of the lumbar spine demonstrating epidural collection at L5 (arrow), enhancing soft tissue within the epidural space from L4-S1 suggestive of phlegmonous change and discitis/osteomyelitis. a Postcontrast sagittal T1-weighted MRI. b Sagittal T2-weighted MRI. c STIR image

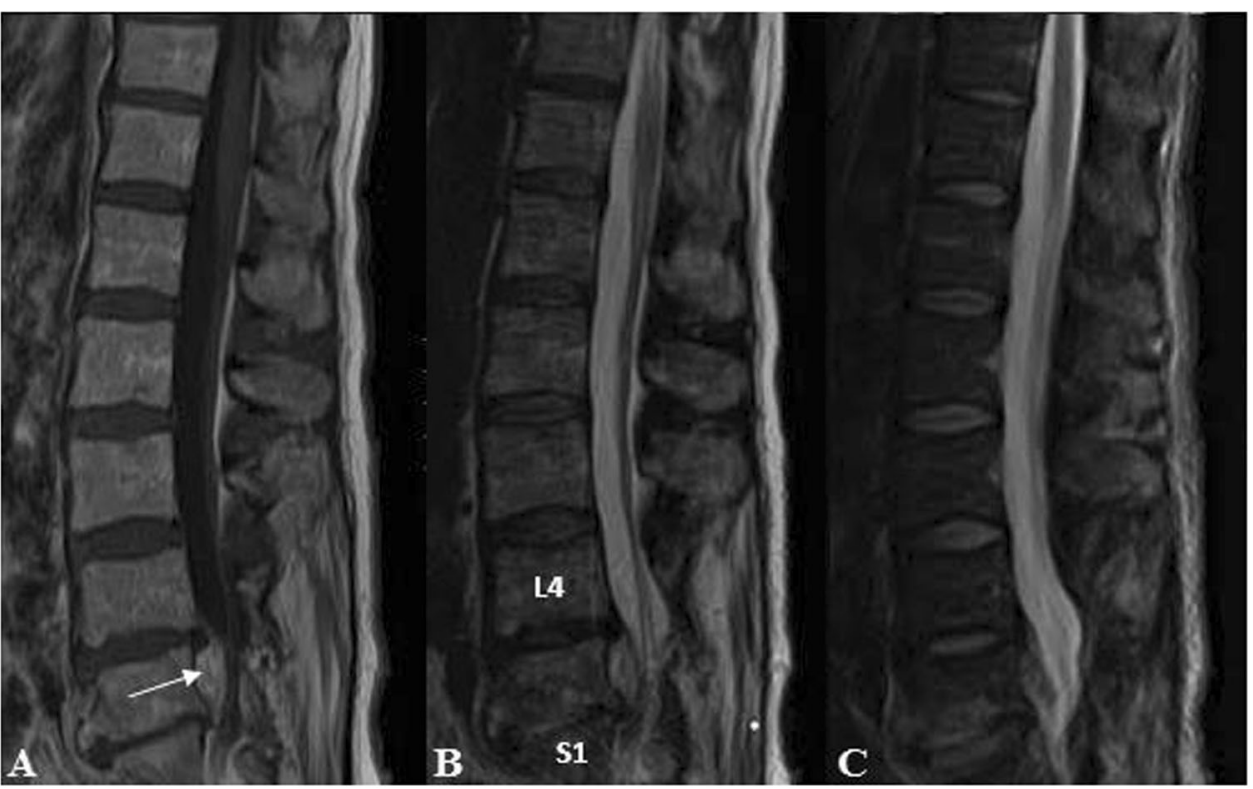

what was thought to be a bacterial SEA. However, cytopathology of the CT-guided, percutaneously drained fluid from the epidural collection was positive for acute angle branching hyphae consistent with Aspergillus fungi, and treatment was initiated with intravenous antifungals (Voriconazole and Micafungin). Given that the patient lacked any neurological deficits or any signs of cauda equina syndrome, spinal decompressive surgery was not initiated at this time. Her condition remained stable over the next 2 days, at which point she received drainage of the right sacroiliac joint abscess and cytopathology was also positive for Aspergillus, indicating that both results were unlikely to be contaminants. Subsequent lumbar MRI performed 11 days later showed significant decrease in size of the epidural abscess, and invasive surgery was deemed unnecessary. Medical management with long-term intravenous Micafungin and Voricanozole was pursued, and patient was discharged to a skilled nursing facility to complete the planned 3-month course. Physical and occupational therapy noted the patient to progress well, returning very near baseline upon discharge.

\section{Literature Review}

Searching the literature we found 26 previously reported cases summarized in Tables 1 and 2 [1-10, 16-18, 20-22, 24-26, 28-32]. These infections were found to occur in patients most commonly in their third to sixth decades of life, but the age of onset can vary at an average of 44 years and a range of 12-66 years. The incidence is much higher in males $(66.7 \%)$. Aspergillus spinal abscess is most prevalent in the thoracic region of the vertebral column, but is also fairly frequently found in the lumbar spine $(53.8 \%$ vs.
$42.3 \%$, respectively). Common symptoms on presentation are pain in the lower back, focal neurological deficits, and fever. MRI and CT myelography were most frequently used for diagnostic imaging. Most common comorbidities were cancer, diabetes mellitus, immunodeficiency, tuberculosis, and renal failure. Complications were disastrous in a number of instances and included intracranial hemorrhage, gastrointestinal hemorrhage, amphotericin B toxicity, meningoencephalitis, and multi-organ failure. Treatment usually entailed a combination of antibiotics and surgical intervention. Only a single case was treated with antibiotics alone [16].

In terms of overall clinical outcomes, death occurred in the majority (52\%), with many dying in the immediate postoperative period or at mean follow-up of 1.8 months following discovery. In another outcome group there was full recovery (26\%) and these patients attained their near preoperative baseline functional level at a mean follow-up of 14.4 months. In a subset of patients there was intermediate recovery $(22 \%)$ of patients, in which minimal resolution of symptoms occurred but who are alive and functional at mean follow-up of 14.8 months.

\section{Discussion}

The most common invasive Aspergillus is Aspergillus fumigatus, but other species have also been described in the literature $[8,34]$. Despite its ubiquitous presence in the environment, invasive infection seldom occurs [24]. In the spine, it most commonly affects the vertebral bodies and intervertebral discs and often results in osteomyelitis [16]. In immunocompromised patients, in particular, Aspergillus 


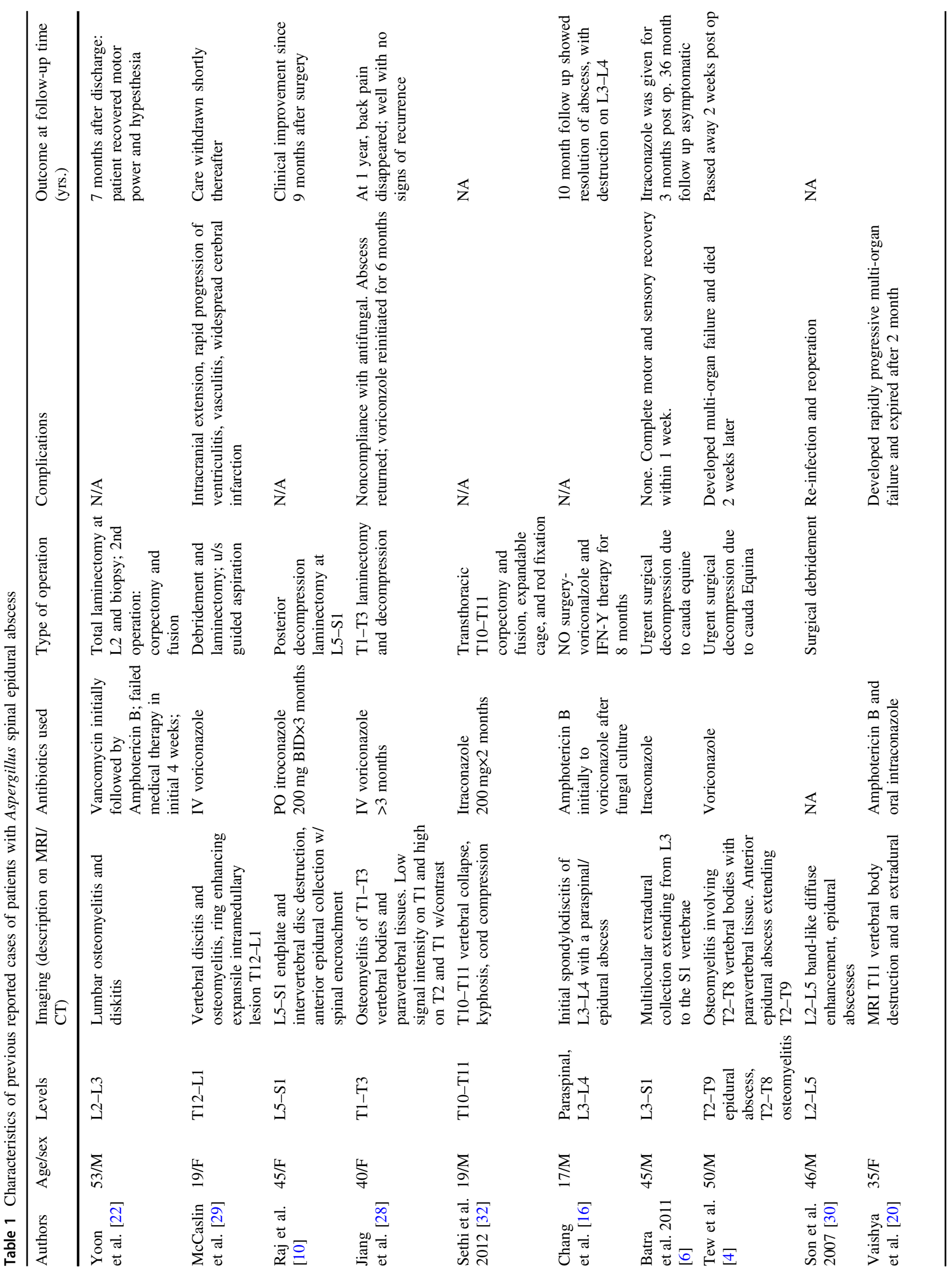




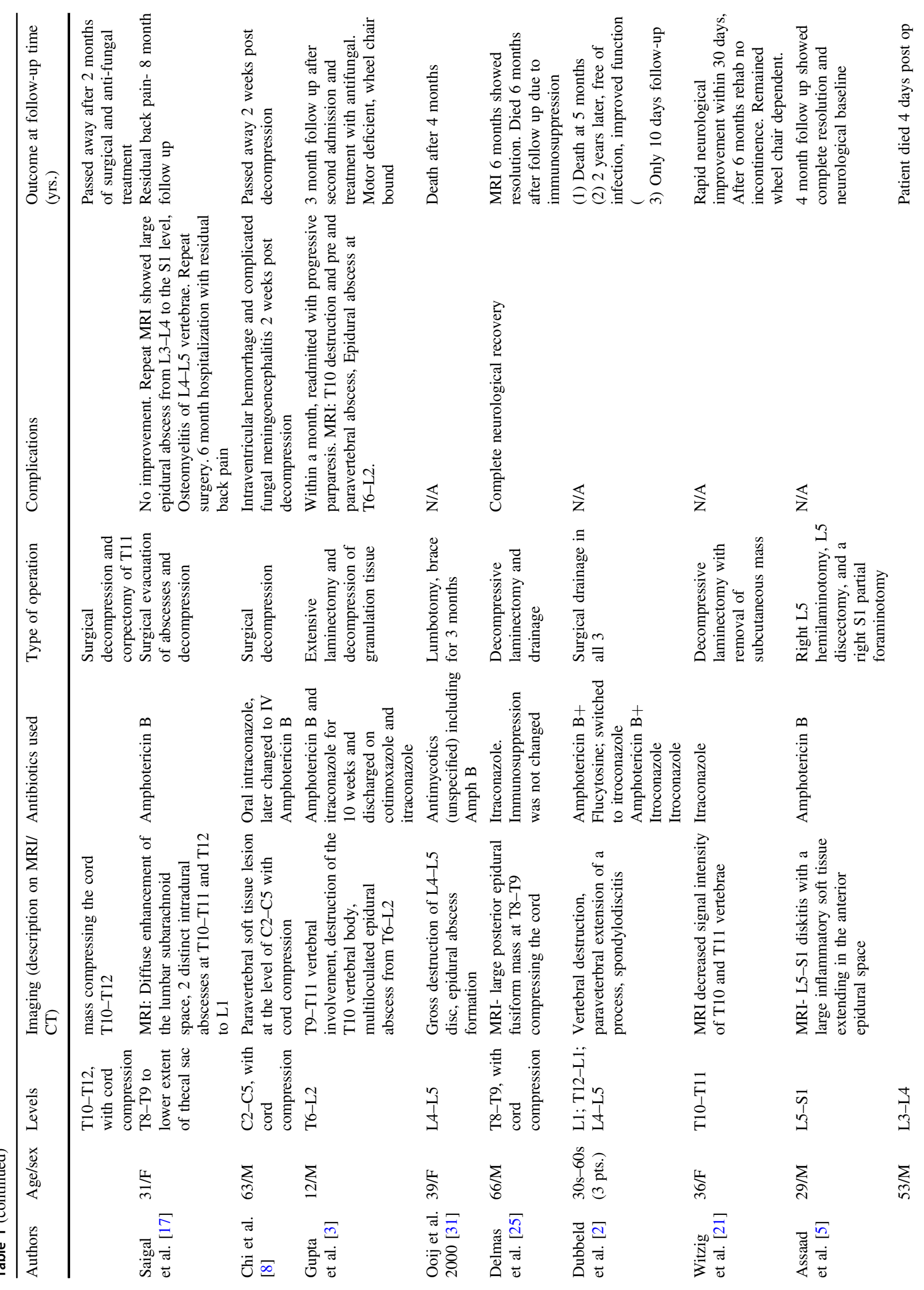




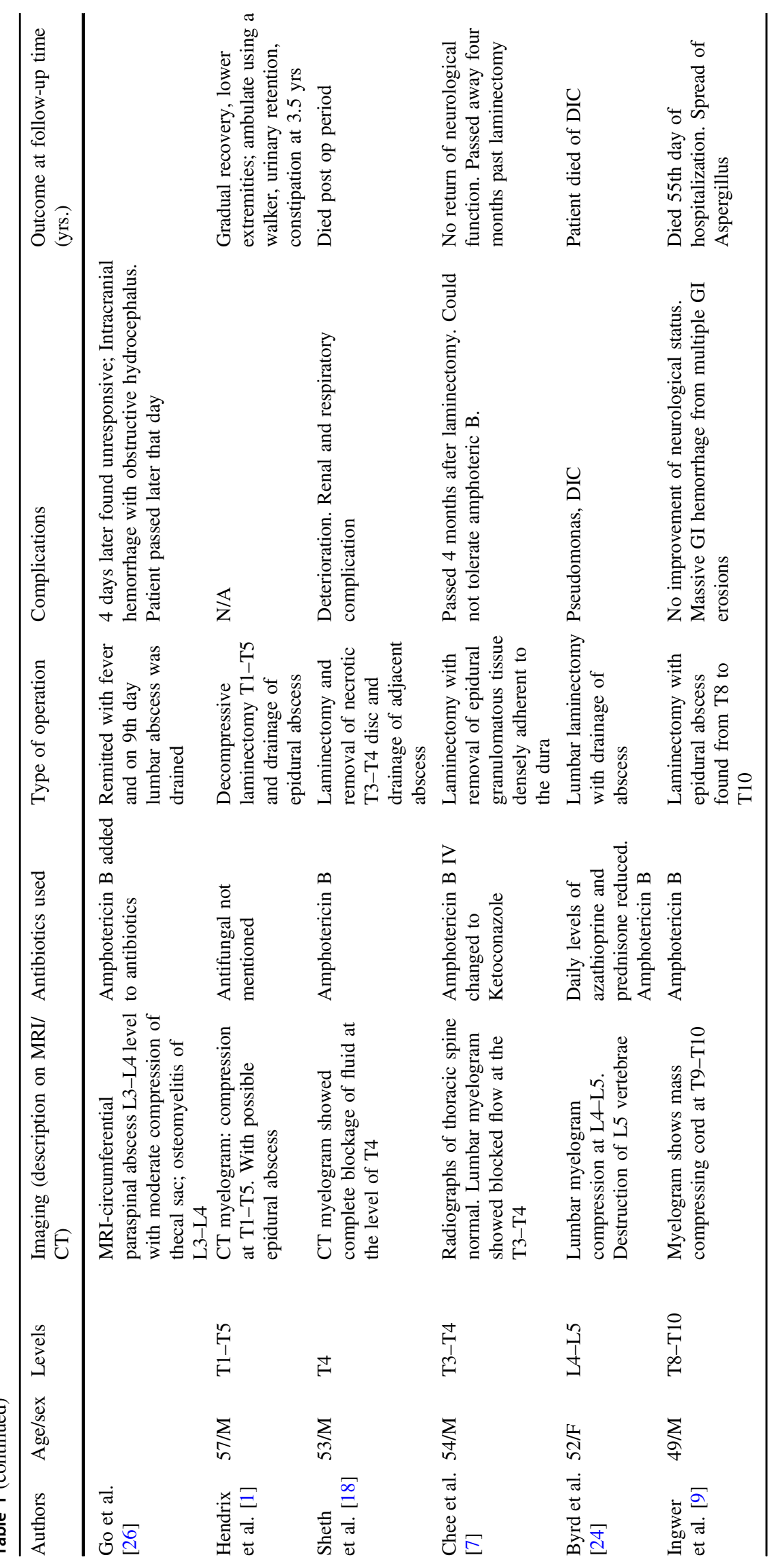


Table 2 Summary of clinical features from previous papers describing patients with aspergillus spinal epidural abscess

\begin{tabular}{|c|c|}
\hline Feature & Result \\
\hline Mean age & 42.3 years \\
\hline Sex & $\begin{array}{l}66.7 \% \text { male } \\
33.3 \% \text { female }\end{array}$ \\
\hline Location & $\begin{array}{l}\text { Thoracic: } 53.8 \% \\
\text { Lumbar: } 42.3 \% \\
\text { Cervical: } 3.8 \%\end{array}$ \\
\hline Comorbidities & $\begin{array}{l}\text {-Immunodeficiency: } 9 \\
\text {-Aspergilloma: } 6 \\
\text {-Cancer: } 5 \\
\text {-Diabetes Mellitus: } 4 \\
\text {-Tuberculosis history: } 3 \\
\text {-Renal failure and/or transplant: } 3 \\
\text {-History of epidural injections: } 2\end{array}$ \\
\hline Complications & $\begin{array}{l}\text {-Readmitted/Reoperation: } 5 \\
\text {-Intracranial extension/hemorrhage: } 4 \\
\text {-Multi-organ failure, DIC, other hemorrhage: } 4 \\
\text {-Amphotericin B toxicity: } 1\end{array}$ \\
\hline \multirow[t]{3}{*}{ Outcome } & $\begin{array}{l}\text {-Outcome Group } 1 \text { - Death }(52 \%) \text { : at mean follow- } \\
\text { up of } 1.8 \text { months (many in the immediate post- } \\
\text { operative period) }\end{array}$ \\
\hline & $\begin{array}{l}\text {-Outcome Group } 2 \text { - Intermediate Recovery ( } 22 \%) \text { : } \\
\text { minimal resolution in symptoms but patient is alive } \\
\text { and functional at mean follow-up of } 13.8 \text { months }\end{array}$ \\
\hline & $\begin{array}{l}\text {-Outcome Group } 3 \text { - Full Recovery }(26 \%) \text { : patients } \\
\text { made a full recovery, near to preoperative baseline at } \\
\text { mean follow-up of } 13.3 \text { months }\end{array}$ \\
\hline
\end{tabular}

can be contracted by inoculation during surgery or other procedures from the surrounding air [5]. A common theme among patients with ASEA is immunosuppression, which can be a result of various conditions, including intravenous drug abuse, corticosteroid therapy, and HIV status [5, 7, 24, 25]. Invasive Aspergillus is particularly prevalent in cases of damaged phagocytic function, as in chronic granulomatous disease [4, 6]. Other patients at risk for Aspergillus infection include those with a history of renal transplant, liver cirrhosis, and bodily prosthetics [4, 8, 25]. Diabetic patients have also reportedly developed ASEA with neutrophil dysfunction a likely contributor [8]. The most common source and route of infection is hematogenous spread from the pulmonary system and/or genitourinary system.

Recently, outbreaks of fungal spinal infections in patients receiving steroidal spinal epidural injections was reported [13, 14]. Smith et al. [15] reported on the outbreak of a series of fungal infections that occurred in patients receiving steroid injections at more than 70 facilities throughout the United States. Besides spinal infections, patients reportedly developed joint infections, meningitis, and strokes in one of the largest described outbreaks in the healthcare arena. The result was 749 cases and 61 deaths [13, 15]. Patients with CNS involvement were more likely to have received a higher dose of methylprednisolone (>80 mg), a translaminar approach epidural injection, and an associated comorbidity such as diabetes mellitus and hypertension [13], with epidural abscess occurring at an estimated 39 days following administration of the steroid.

Similar to other causes of infection of the spine, typical symptoms on presentation include pain in the lower back and paresthesia of the lower extremities. Other common symptoms include pain in the lower extremities, urinary retention and fevers (Table 1). Because of the length of time required for the symptoms to become debilitating and the lack of specificity of the symptoms, diagnosis may be delayed as much as 5-7 months from the onset of the earliest symptom [7]. A recent paper alludes to the danger of late recognition with resulting disastrous consequences such as intramedullary involvement and intracranial spread [29]. In addition, not every patient presents with fever and neurological deficits, demonstrating the need for proper diagnostic testing [25]. The erythrocyte sedimentation rate (ESR) is generally increased and allows for a way for assessment at follow-up and treatment response [3].

Presently the gold standard for confirmatory diagnosis is histopathology and culture of the offending microorganism $[3,4]$. However, obtaining a definitive culture can take many weeks, delaying diagnosis [4]. Because of the limitations in obtaining such a definitive diagnosis, methods with molecular biology have been utilized, including enzyme-based and PCR-based assays and are characterized by their earlier diagnostic results [4]. However, these are limited by either lower sensitivity or higher false positives. The primary imaging modalities used to determine the diagnosis are CT myelography and MRI (Table 2). Computerized tomography delineates bone and soft tissue damage in the surrounding area, but MRI is a better modality for diagnosing the phlegmonous lesion, although MRI results may vary for ASEA [4, 8, 33]. Characteristically, SEA of the Aspergillus type can be differentiated by its shape, which is irregular and thick-walled and two other features on imaging: band-like subchondral T2 hypointensity and vertebral endplate irregularities on T1 [17] (see Fig. 2). Importantly, image-guided biopsy can be a powerful diagnostic and therapeutic tool. This was demonstrated impressively in our patient.

The primary mode of treatment in patients listed in Table 2 was a combination of antifungals and surgical decompression and debridement. Standardizing management is difficult for this condition, though surgical drainage combined with intravenous antifungal therapy seems to be the most common approach [6, 8, 16]. While amphotericin B was frequently used in earlier reported cases, it is nephrotoxic and has reportedly been found to be ineffective in 
some cases of invasive Aspergillus because of lack of entry into bone $[3,6,9,16]$. Second generation triazoles, like Voriconazole, have been shown to be more efficacious and less toxic for the patient on long-term treatment [16]. Flucytosine may also be a good addition to the treatment regimen for vertebral infections concurrent with abscess $[2$, $6,16]$. Treatment must be administered until symptoms resolve with concordant clearance of the abscess on imaging and continued for multiple months [8]. In addition, symptomology dictates the protocol for surgery-those lacking neural compression may be treated medically, but only with very close monitoring $[7,28]$.

Unfortunately, despite treatment, the condition is associated with a high long-term mortality rate, and many patients have persistent symptoms, such as paraplegia and various serious complications ranging from hemorrhage to multi-organ failure. In fact, as seen in Table 2, more than half the patients died and only 7 patients made a complete recovery (one dying soon after). In addition, 5 patients made partial recovery, but remained wheel chair bound and two others had significant residual symptoms. These dismal findings highlight the need for better recognition of this disease entity, improved treatment modalities, and safer interventions. This becomes even more important from a public health standpoint during times of infectious disease outbreaks, such as the recent series of fungal infections that occurred in mid-late 2012 [13-15]. The high mortality rate is likely for a number of reasons such as many patient comorbidities (immunosuppression being at the top of the list) and patient status on presentation to the hospital (those with a poor functional status likely having a worse outcome). It is interesting to note the finding of hemorrhage (i.e. intracranial or gastrointestinal) as one of the recurring complications of this unique infection. As to whether surgery correlates with a higher mortality rate is difficult to determine as almost all previous cases were treated via surgery and many had high mortality while others had a better outcome.

In our case, the patient was treated successfully without amphotericin B, eliminating the unpleasant and potentially risky side effects. Additionally, she was treated conservatively without surgical intervention. This was decided based on lack of neurological deficits on presentation and significant improvement of the abscess on MRI following administration of antifungal therapy. This supports the view that effective treatment for ASEA and osteomyelitis should be centered on systemic antifungal therapy, with surgical intervention acting as a supplementary role to ensure complete clearance of the infection [3, 19, 28]. Voriconazole and Micafungin were used and the authors report an adequate outcome with this combination of antifungals. Being a fungal cell wall inhibitor, Micafungin, part of the Echinocandin class of antifungals, has a relatively benign side effect profile with very minimal toxicity [34-36]. Duration of antifungal treatment should be at least 3-6 months or a longer length of time [16, 27]. While surgical excision should be considered in cases with cord compression and for relief of neurological symptoms and those that don't resolve with drug treatment alone $[3,6,23$, 28]. Additionally, we recommend surgery for large collections, patients with neurological symptoms, especially those that are progressive, when diagnosis is very uncertain, and there is lack of response to medical management alone. Location of the epidural collection is also of significance, with more invasive management to likely be undertaken on cervical and thoracic abscesses than those in the lumbar spine.

\section{Conclusion}

We describe a patient with a history of chronic back pain treated with spinal epidural injections who presents with symptoms of a probable spinal infection and was subsequently diagnosed with ASEA. She was successfully treated with conservative, nonsurgical management, and we report success with a Voriconazole and Micafungin combination. Confirmatory diagnosis has to be with histopathological and microbiological analysis, though earlier diagnosis is crucial and enzyme/PCR assays are alternatives. Understanding the management of aspergillus is also essential from a public health standpoint, as outbreaks involving fungal spinal infections have been reported in patients receiving invasive spinal interventions.

\section{Compliance with ethical standards}

Conflict of interest The authors declare that they have no conflict of interest.

\section{References}

1. Hendrix WC, Arruda LK, Platts-Mills TA, Haworth CS, Jabour R, Ward GW. Aspergillus epidural abscess and cord compression in a patient with aspergilloma and empyema. Am Rev Respir Dis. 1992;145:1483-6.

2. Dubbeld P, van Oostenbrugge RJ, Twinjstra A, Schouten HC. Spinal epidural abscess due to Aspergillus infection of the vertebrae: report of 3 cases. Neth J Med. 1996;48:18-23.

3. Gupta PK, Mahapatra AK, Gaind R, Bhandari S, Musa MM, Lad SD. Aspergillus spinal epidural abscess. Pediatr Neurosurg. 2001;35:18-23.

4. Tew CW, Han FC, Jureen R, Tey BH. Aspergillus vertebral osteomyelitis and epidural abscess. Singapore Med J. 2009;50: e151-154.

5. Assaad W, Nuchikat PS, Cohen L, Esguerra JV, Whittier FC. Aspergillus discitis with acute disc abscess. Spine. 1994;19:2226-9. 
6. Batra S, Arora S, Meshram H, Khanna G, Grover SB, Sharma VK. A rare etiology of cauda equina syndrome. J Infect Dev Ctries. 2011;5:79-82.

7. Chee YC, Poh SC. Aspergillus epidural abscess in a patient with obstructive airway disease. Postgrad Med J. 1983;59:43-45.

8. Chi CY, Fung CP, Liu CY. Aspergillus flavus epidural abscess and osteomyelitis in a diabetic patient. J Microbiol Immunol Infect. 2003;36:145-8.

9. Ingwer I, McLeish KR, Tight RR, White AC. Aspergillus fumigatus epidural abscess in a renal transplant recipient. Arch Intern Med. 1978;138:153.

10. Raj KA, Srinivasamurthy BC, Nagarajan K, Sinduja MG. A rare case of spontaneous Aspergillus spondylodiscitis with epidural abscess in a 45-year-old immunocompetent female. J Craniovertebr Junction Spine. 2013;4:82-84.

11. Thurnher MM, Olatunji R. Infections of the spine and spinal cord. Handbook Clin Neurol. 2016;136:717-31.

12. Duarte RM, Vaccaro AR. Spinal infection: state of the art and management algorithm. Eur Spine J. 2013;22:2787-99.

13. Chiller TM, Roy M, Nguyen D, Guh A, Malani AN, Latham R, et al. Multistate Fungal Infection Clinical Investigation Team Clinical findings for fungal infections caused by methylprednisolone injections. N Engl J Med. 2013;369:1610-9.

14. Kainer MA, Reagan DR, Nguyen DB, Wiese AD, Wise ME, Ward J, et al. Tennessee Fungal Meningitis Investigation Team Fungal infections associated with contaminated methylprednisolone in Tennessee. N Engl J Med. 2012;367:2194-203.

15. Smith RM, Schaefer MK, Kainer MA, Wise M, Finks J, Duwve J, et al. Multistate Fungal Infection Outbreak Response Team Fungal infections associated with contaminated methylprednisolone injections. N Engl J Med. 2013;369:1598-609.

16. Chang HM, Yu HH, Yang YH, Lee WI, Lee JH, Wang LC, et al. Successful treatment of Aspergillus flavus spondylodiscitis with epidural abscess in a patient with chronic granulomatous disease. Pediatr Infect Dis J. 2012;31:100-1.

17. Saigal G, Donovan Post MJ, Kozic D. Thoracic intradural Aspergillus abscess formation following epidural steroid injection. Am J Neuroradiol. 2004;25:642-4.

18. Sheth NK, Varkey B, Wagner DK. Spinal cord aspergillus invasion--complication of an aspergilloma. Am J Clin Pathol. 1985;84:763-9.

19. Tang TJ, Janssen HL, van der Vlies CH, de Man RA, Metselaar HJ, Tilanus HW, et al. Aspergillus osteomyelitis after liver transplantation: conservative or surgical treatment? Eur J Gastroenterol Hepatol. 2000;12:123-6.

20. Vaishya S, Sharma MS. Spinal Aspergillus vertebral osteomyelitis with extradural abscess: case report and review of literature. Surg Neurol. 2004;61:551-5.
21. Witzig RS, Greer DL, Hyslop NE Jr. Aspergillus flavus mycetoma and epidural abscess successfully treated with itraconazole. J Med Vet Mycol. 1996;34:133-7.

22. Yoon KW, Kim YJ. Lumbar Aspergillus osteomyelitis mimicking pyogenic osteomyelitis in an immunocompetent adult. Br J Neurosurg. 2015;29:277-9.

23. Bridwell KH, Campbell JW, Barenkamp SJ. Surgical treatment of hematogenous vertebral Aspergillus osteomyelitis. Spine. 1990;15:281-5.

24. Byrd BF, Weiner MH, McGee ZA. Aspergillus spinal epidural abscess. JAMA. 1982;248:3138-9.

25. Delmas Y, Merville P, Dousse V, Dervau-Durieux L, Morel D, Potaux L. A renal transplant recipient with acute paraparesis due to an Aspergillus epidural abscess. Nephrol Dial Transplant. 1997; 12:2185-7.

26. Go BM, Ziring DJ, Kountz DS. Spinal epidural abscess due to Aspergillus sp in a patient with acquired immunodeficiency syndrome. South Med J. 1993;86:957-60.

27. Horn D, Sae-Tia S, Neofytos D. Aspergillus osteomyelitis: review of 12 cases identified by the Prospective Antifungal Therapy Alliance registry. Diagn Microbiol Infect Dis. 2009;63:384-7.

28. Jiang Z, Wang Y, Jiang Y, Xu Y, Meng B. Vertebral osteomyelitis and epidural abscess due to Aspergillus nidulans resulting in spinal cord compression: case report and literature review. J Int Med Res. 2013;41:502-10.

29. McCaslin AF, Lall RR, Wong AP, Lall RR, Sugrue PA, Koski TR. Thoracic spinal cord intramedullary aspergillus invasion and abscess. J Clin Neurosci. 2015;22:404-6.

30. Son JM, Jee WH, Jung CK, Kim SI, Ha KY. Aspergillus spondylitis involving the cervico-thoraco-lumbar spine in an immunocompromised patient: a case report. Korean J Radiol. 2007;8:448-51.

31. van Ooij A, Beckers JM, Herpers MJ, Walenkamp GH. Surgical treatment of aspergillus spondylodiscitis. Eur Spine J. 2000;9:75-9.

32. Sethi S, Siraj F, Kalra K, Chopra P. Aspergillus vertebral osteomyelitis in immunocompetent patients. Indian $\mathbf{J}$ Orthop. 2012;46:246-50.

33. Kwon JW, Hong SH, Choi SH, Yoon YC, Lee SH. MRI findings of Aspergillus spondylitis. AJR Am J Roentgenol. 2011;197: W919-923.

34. Wang JL, Chang CH, Young-Xu Y, Chan KA. Systematic review and meta analysis of the tolerability and hepatotoxicity of antifungals in empirical and definitive therapy for invasive fungal infection. Antimicrob Agents Chemother. 2010;54:2409-19.

35. Ashley ES, Lewis R, Lewis J, Martin C, Andes D. Pharmacology of Systemic Antifungal Agents. Clin Infect Dis. 2006;43:S28-39.

36. Lewis RE. Current concepts in antifungal pharmacology. Mayo Clin Proc. 2011;86:805-17. 